\title{
Smooth pursuits decrease balance control during locomotion in young and older healthy females
}

Neil M. Thomas, 1 困

Email neil.thomas@uni.cumbria.ac.uk

Susan Dewhurst, 1

Theodoros M. Bampouras, 1

Tim Donovan, 1

Andrea Macaluso, 2

Giuseppe Vannozzi, 2

1 Department of Medical and Sport Sciences, Active Ageing Research Group, University of Cumbria, Lancaster, LA1 4DH UK

2 Department of Movement, Human and Health Science, University of Rome Foro Italico, Piazza Lauro de Bosis 6, 00135 Rome, Italy

\section{Abstract}

Dynamic balance control-characterised as movement of the trunk and lower limbs - was assessed during smooth pursuit and saccadic eye movements in ten young $(22.9 \pm 1.5$ years $)$ and ten older (72.1 \pm 8.2 years) healthy females walking overground. Participants were presented with visual stimuli to initiate eye movements, and posture and gaze were assessed with motion analysis and eye tracking equipment. The results showed an increase in medial/lateral (ML) trunk movement (C7: $p=0.012$; sacrum: $p=0.009$ ) and step-width variability ( $p=0.052$ ) during smooth pursuits compared to a fixed target, with no changes for saccades. The elders 
demonstrated greater ML trunk movement (sacrum: $p=0.037$ ) and step-width variability $(p=0.037)$ than the younger adults throughout, although this did not interact with the eye movements. The findings showed that smooth pursuits decreased balance control in young and older adults similarly, which was likely a consequence of more complicated retinal flow. Since healthy elders are typically already at a postural disadvantage, further decreases in balance caused by smooth pursuits are undesirable.

AQ1

\section{Introduction}

Vision is important for balance control during locomotion. Experiments manipulating the visual field of young adult treadmill walkers, for example, have resulted in forward and backward trunk lean (Logan et al. 2010) and increased medial/lateral (ML) trunk movement and step-width variability (Warren et al. 1996; McAndrew et al. 2010). Step-width variability is of particular interest as it is linked to control of the bodies centre of mass (COM) on a step-to-step basis and is important for maintaining balance (Bauby and Kuo 2000). These findings were thought to result from the central nervous system (CNS) detecting changes to the visual field and adjusting posture (albeit in error) accordingly.

Visual sensing of the external environment and self-motion within it occurs at the retina. In a 3D world, patterns of light reflected off structures reaching the retina create an optic array. If the observer moves, it changes the structure of the array about a point of central observation (Gibson 1950). Such changes in patterns of light which flow across the retina are thought to be interpreted to estimate body position (Warren et al. 1996; Logan et al. 2014). However, eye movements can change the structure of the array, and flow patterns on the retina can be a combination of those caused by self-motion in addition to those caused by eye movements (Warren and Hannon 1990; Lappe and Hoffmann 2000). The CNS must, therefore, solve a source 
separation issue between the two when judging self-motion (DeAngelis and Angelaki 2012).

Despite investigations about how humans control their direction heading during eye movements (Royden et al. 1994), studies manipulating visual flow and assessing balance during walking have not considered eye movements. If changes to flow caused by eye movements affect how humans interpret the optic array, it may in turn affect balance control.

The nature of changes to balance control would likely depend on the type of eye movement. For instance, visually fixating a stationary object straight ahead would cause radial flow from forward progression, and this would emanate from the central point of observation (Warren and Hannon-1990Lappe and Hoffman 2000). Such flow may be considered useful for balance control since it provides a stable reference frame [assuming healthy vestibulo-ocular (VOR) and vestibulo-colic reflexes] from which self-motion with respect to the vertical can be determined. Conversely, tracking an object in horizontal motion would cause horizontal flow from eye rotation in addition to radial flow from forward progression. The resulting pattern would resemble a curved movement with a shifting focus of expansion (Warren and Hannon 1990; Lappe and Hoffmann 2000). Moreover, although the object of fixation would appear stabilised on the fovea, the background information would become blurred (Kowler 2011). This added complexity may cause difficulty when estimating self-motion, thus decreasing balance control. Saccades are another kind of eye movement used during walking. These are rapid shifts of gaze from one region to another (Kowler 2011). However, because saccades are a series of fixations separated by rapid intervals, unless the saccades were to an extreme displacement and/or with unnaturally high frequency, the stable reference frame provided by fixation should be preserved.

Of interest when considering the above are older individuals. Elders can be more sensitive to ML perturbations of the visual field during walking resulting in greater reduction of trunk stabilisation and 
increased step-width variability when compared to younger adults (Franz et al. 2015). Further, it is thought the ageing CNS relies on vision more for balance control because of vestibular and musculoskeletal sensory declines (Bugnariu and Fung 2007; Yeh et al. 2014). Therefore, because elders cannot decompose retinal flow as effectively as young adults, and can be more reliant on visual information, any decrease in balance control caused by smooth pursuits may be more profound in this age group. One study found a comparable increase in postural sway during smooth pursuits between young and older adults during standing (Thomas et al. 2016). However, because the biomechanical constraints and nature of visual flow during walking are so different to standing, further investigation is warranted.

Therefore, the present study assessed balance control during smooth pursuits and saccades in healthy young and older females walking overground. It was hypothesised that: (a) smooth pursuits would increase medial/lateral (ML) trunk movement and step-width variability; (b) this would be more profound in older adults; (c) saccades would maintain balance compared to fixating a stable target.

\section{Materials and methods}

\section{Participants}

Ten young (mean \pm SD: $22.9 \pm 1.5$ years; $1.7 \pm 0.06 \mathrm{~m}$; $59.5 \pm 7.2 \mathrm{~kg}$ ) and ten older (mean \pm SD: $72.1 \pm 8.2$ years; $1.6 \pm$ SD $0.03 \mathrm{~m}$; $57.3 \pm 5.6 \mathrm{~kg}$ ) healthy females participated in the study. The elders were interviewed initially to determine suitability for the study. All participants adhered to inclusion criteria previously outlined (Thomas et al. 2016). In short, they had no known musculoskeletal or neurophysiological conditions which could affect normal balance during standing and walking. All participants had an uncorrected visual acuity (without glasses or contact lenses) $\geq 20 / 100$ and were able to ambulate in the community without visual correction. The investigation was carried out in accordance with the recommendations of the University of Cumbria ethical principles and guidelines for research involving human subjects, and all procedures, information to 
the participants and participant consent forms were approved by the University of Cumbria Research Committee. All subjects gave written informed consent in accordance with the Declaration of Helsinki.

\section{Equipment}

Visual scenes were projected (Sanyo PLC-XU74, Tokyo, Japan) onto a $3.2 \times 2.4 \mathrm{~m}$ screen on the wall of the laboratory. Participants wore eye tracking glasses (Tobii Glasses 2 Eye Tracker, Tobii Technology, Danderyd, Sweden) which have a one-point calibration procedure, autoparallax compensation and slippage compensation allowing for persistent calibration throughout testing with no loss of data aside from blinking. A 7 camera Vicon system (MX3, Oxford, UK; sampling frequency $100 \mathrm{~Hz}$ ) recorded three-dimensional positions of eight passive reflective markers located at the left and right front and back head, C7, sacrum, and left and right heel anatomical landmarks of each participant. A custom-made contact mat was used to initiate visual stimulus movement (see "Experimental protocol”).

\section{Visual stimuli}

Visual stimuli were programmed with Psychopy stimuli presentation software (Peirce 2007). The visual target presented was a light blue circle displayed over a black background. Each participant could see the target at all times during testing. Three experimental conditions were implemented: stationary gaze fixation (FIX), smooth pursuit (PUR) and saccadice (SAC). For FIX, the target remained in the centre of the screen at eye level. During PUR, the target displaced from the centre of the screen in the horizontal direction to a defined threshold of visual angle (described below) before returning to the centre of the screen with a frequency of $0.33 \mathrm{~Hz}$ (Glasawer et al. 2005; Laurens et al. 2010). The target moved randomly left or right on each oscillation, which had no bearing or relation to the participants side dominance. This choice reflects spontaneous tracking movements occurring in everyday activities (Kowler 2011). For SAC, the same protocol was implemented. However, the target disappeared from the centre of the screen and reappeared at the defined threshold 
stimulating a saccadic eye movement, and then disappeared and reappeared back at the centre.

The stimuli were programmed in degrees of visual angle enabling standardisation between laboratories/experiments. It was considered to update the size and displacement of the visual target relative to each participant as they progressed along the walkway. This would have maintained a constant degree of visual angle for the size of the target, and visual angle change for the displacement of the target. However, this would have made the target appear to move away from the observer as they progressed forward. In everyday life, objects do not typically reduce in size as an observer approaches. Likewise, objects moving across the field of vision such as a passing pedestrian often maintain a linear heading. The magnitude of the tracking movement is such a case is thus always dependent on how far the observer is from a given visual target. It was decided, therefore, not provide real-time adjustments. As such, the size of the visual target relative to each participant corresponded to $1^{\circ}$ at the start of the data capture area, and $2^{\circ}$ at the end. The displacement of the target (left or right) in the horizontal direction corresponded to $6^{\circ}$ at the start of the data capture area and $12^{\circ}$ at the end.

\section{Experimental protocol}

Five trials for each condition (FIX, PUR and SAC) were completed. The conditions were sorted randomly and segregated into 3 blocks of 5 trials. Each block was separated by 2 min of rest. The participants walked overground on a flat level walkway in the laboratory for $7.5 \mathrm{~m}$. The walkway consisted of a $2.5 \mathrm{~m}$ entry area to achieve a steady-state velocity, which has previously been recommended for older individuals (Lindemann et al. 2008), a $4 \mathrm{~m}$ data capture area where balance control was assessed, and a $1 \mathrm{~m}$ exit area (Fig. 1). At least 2.5 strides of data were collected from each participant during each trail which totalled at least 12.5 strides for each participant in each condition. FIX was presented at the beginning of the entry area before the participants set off during all trials. On the first heel strike on entering the data capture area, which was arranged to be in the first $30 \mathrm{~cm}$, visual target 
movement or no movement depending on the condition was initiated by the custom-made contact mat.

\section{Fig. 1}

Walkway consisting of entry area (a); contact mat (b); data collection area (c); exit area (d); and projection screen (e). Asterisk distance between walkway and projection screen not to scale

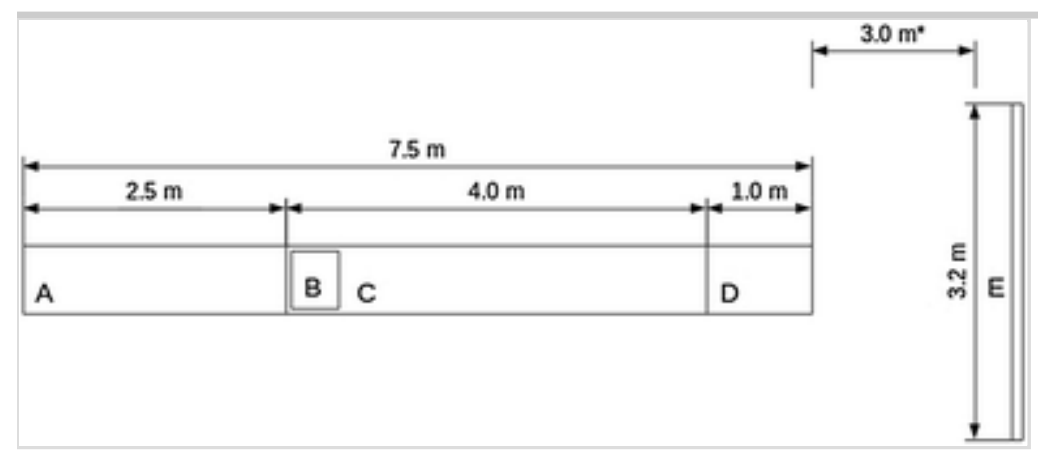

Participants were instructed to fixate their gaze on the visual target at all times. If it moved, they should follow it with their eyes only making sure not to rotate or tilt their head. Gaze shifts less than $15^{\circ}$ are commonly achieved without rotation of the head (Hallet 1986), and eye movements up to $35^{\circ}$ have been performed whilst minimising head movements (Paquette and Fung 2011). This accommodates the maximum target displacement of $12^{\circ}$ in the present investigation. Head rotations during testing were assessed to ensure any changes in the outcome measures were not a result of head movements corresponding to the direction of the visual target movement.

\section{Method considerations}

The visual stimuli and experimental set-up aimed to replicate as closely as possible eye movements used in everyday life and their changes to flow whilst standardising eye movement velocity and displacement. Using a virtual reality environment with 3D cues, which would generate similar retinal flow patterns as to walking through a room, was considered. However, this would require treadmill walking which for reasons discussed below was not appropriate. Instead, the 2D visual target was projected at the wall of the laboratory, and as such 
there would have been visual flow generated from the rest of the room as the participants walked forward, e.g. from the walls running adjacent to the walkway. With regard to the target, this just provided a visual fixation point — during a smooth pursuit it is the background visual information (the rest of the room in the present experiment) that becomes more difficult to interpret, and this is what has previously been suggested to affect standing postural control (Glasawer et al. 2005; Laurens et al. 2010; Thomas et al. 2016). The object of fixation is, therefore, not of particular concern.

With regard to locating the target at eye level and in the centre of the visual field, although humans have been shown to fixate on points at which they will step around a second or so before stepping on them (Patla and Vickers 2003), walking humans also locate their gaze centrally along the horizontal and vertical relative to the direction heading. In other words, toward a heading point (Foulsham et al. 2011). Moreover, they often fixate on fixed and moving objects including near and far standing and moving humans (Foulsham et al. 2011) and things located in the field of vision such as posters affixed to walls (Dowiasch et al. 2015). One study found that of 133 pedestrians, a walker fixated $83 \%$ of them at least once whilst navigating a university campus (Foulsham et al. 2011). This coupled with a previously documented visual attentional bias towards people's faces and eyes (Birmingham et al. 2008) suggests that gaze is often allocated at eye level in front of the observer, and sometimes on moving targets.

Finally, the number of recommended strides for assessing gait variability has ranged between 5 and 8 to the order of hundreds whilst dual tasking in older individuals (Owings and Grabiner 2004; Hollman et al. 2010). Additionally, separating data collection into a series of stop-start walks as in the present experiment can increase lower-limb variability when compared to one continuous walk (Paterson et al. 2009). However, measuring gait for long duration walking would typically require a treadmill which has been shown to significantly alter gait (Dingwell et al. 2001). Further, replicating normal retinal flow patterns on a treadmill would require virtual reality. In addition to 
the limited availability of such a set up, virtual reality has also been shown to alter gait compared to normal conditions, and may even cause instability in healthy subjects (Hollman et al. 2006,2007). The present investigation, therefore, placed emphasis on repeated short overground walks. First, this more closely replicates everyday walking (Orendurff et al. 2008) in a more familiar way for elders (Wass et al. 2005; Schellenbach et al. 2010). Second, it was important to assess immediate effects of visual stimulus onset. In everyday life eye movements can be initiated spontaneously, and objects of interest may not be observed for many continuous strides. This was important considering the CNS can re-weight its use of vision over longer time frames (Allison et al. 2006) and short-term effects may have gone unnoticed during longer walks.

\section{Data analysis}

Raw marker data were extracted using the Biomechanical Toolkit Python bindings (Barre and Armand 2014) and analysed offline (Scipy, scientific computing tools for Python). Marker trajectories were low-pass filtered using a fourth order zero-phase Butterworth filter with a cutoff frequency of $10 \mathrm{~Hz}$. Heel strike events were determined based on the position of the heel marker in relation to the Sacrum marker (Zeni et al. 2008). In short, the $y$ coordinate of the Sacrum marker at each time frame was subtracted from the $y$ coordinate of each heel marker at the corresponding time frame, and peaks in the resulting time series which represent heel strikes determined. This method has been shown to estimate overground heel strike events to within $0.0021 \mathrm{~s}$ of gold standard force platform measurements.

Trunk movement 
Movement of the lower and upper trunk in the ML direction was quantified as the root mean square (RMS) of the ML component of the C7 and Sacrum markers, where $N=$ number of data points and $n=1$, $\ldots, N$ :

$$
\mathrm{RMS}=\sqrt{\frac{1}{N} \sum n^{2}}
$$

Trunk lean was defined as the inclination angle of the trunk with respect to the vertical axis, which was calculated from the inverse tangent of the distance between the C7 and Sacrum markers in the ML axis divided by the same distance in the vertical axis. RMS of the resulting time series was then computed. The present experiment focused on the ML axis for trunk kinematics as this is sensitive to the visual component of balance control (Warren et al. 1996; McAndrew et al. 2010).

\section{Lower limbs}

Step-width was defined as the ML distance between heel markers at heel strike. Mean and coefficient of variation (CV) where $\mathrm{SD}=$ standard deviation:

$$
\mathrm{CV}=\frac{\mathrm{SD}}{\text { mean }} \times 100
$$

was then calculated for step-width across successive steps (Brach et al. 2005; McAndrew et al. 2010; Franz et al. 2015).

\section{Head rotations}

The four head markers were used to construct a head segment. Then, rotation matrices were calculated between consecutive frames and converted to Euler angles expressed in degrees of yaw rotation about the vertical. This corresponds to the direction of the visual target movement. RMS of the head rotation time series was then computed.

Gaze fixations 
Where participants were looking in relation to the target was assessed to ensure the protocol was completed accurately. Gaze data (sampled at $50 \mathrm{~Hz}$ ) was filtered with a Tobii I-VT fixation filter to yield gaze fixations (window length $20 \mathrm{~ms}$; threshold $30 \% \mathrm{~s}^{-1}$ ). 2D video sequences consisting of the participant's point of view of each visual scene superimposed with their gaze fixations was exported. Position of the target and the position of each gaze fixation as $x$ and $y$ coordinates on the 2D video frame was then determined using a motion tracking algorithm (OpenCV Python libraries). The resultant coordinate time series for each was then calculated. Data before stimulus onset and at the end of the data collection area was removed in accordance with the motion capture data. Where no gaze data were sampled to due blinking, the target coordinate at the corresponding time frame was removed (Thomas et al. 2016). Pearson correlation coefficients were then calculated between the coordinate time series of the target and that of the gaze fixations, and finally RMS of gaze subtracted from the target position (RMS gaze error) throughout each video sequence.

Reliability of the tracking procedure used to determine the coordinates of the target and the gaze fixations was assessed by re-tracking all of the video sequences and computing the CV between gaze error RMS results. A CV of $0 \%$ indicated perfect reliability throughout.

\section{Statistical analysis}

The mean or median of the 5 trials for each participant in each condition was used for statistical analysis of the relevant outcome measures depending on a normal or non-normal distribution of the raw data. Condition ( $3 \times$ visual scenes) and age (young and older) were considered as two independent factors. The effect of these two factors on C7 and sacrum RMS; trunk lean RMS; step-width mean and CV; head rotation RMS; correlation coefficients between the target coordinates and gaze fixation coordinates; and gaze error RMS were examined with a two way (condition $\times$ age) mixed analysis of variance (ANOVA). Where the averages/medians of the trials for each outcome measure departed from normality, effects were re-examined using robust mixed ANOVAs based on trimmed means (Field et al. 2012). 
Post hoc analyses included $t$ tests or Wilcoxon signed-rank tests with Bonferroni corrections. Finally, where significant differences were found ( $p \leq 0.05$ ), Hedges' $g_{\mathrm{av}}$ effect sizes were calculated (Lakens 2013). Common indicative thresholds for effect sizes are small (0.2), medium (0.5) and large (0.8). Statistical results were interpreted in the context of strength of evidence against the null hypotheses, which was determined by the magnitude of the $p$ values (smaller values indicate stronger evidence), magnitude of effect sizes, and 95\% confidence intervals. Statistical analyses were performed with the R software package.

\section{Results}

\section{Trunk movement}

C7 and Sacrum RMS along the ML direction are shown in Fig. 2 and Fig. 3. C7 RMS showed a main effect of condition $\left(F_{2,36}=4.71\right.$, $p=0.015)$. Post hoc comparisons revealed larger C7 RMS during PUR compared to FIX ( $\left.p=0.012, g_{\mathrm{av}}=0.32\right)$, andbut no change for SAC relative to FIX. C7 RMS showed no main effect of age or interaction effect between condition and age.

Fig. 2

C7 RMS in the ML direction in young $(n=10)$ and older $(n=10)$ participants during different eye movements. Fix stationary gaze fixation, Pur smooth pursuit, Sac Saccadice. Data are displayed as means and 95\% confidence intervals in bold, and medians and lower and upper quartiles with Tukey style whiskers (outliers plotted separately). Asterisk significant difference between conditions 


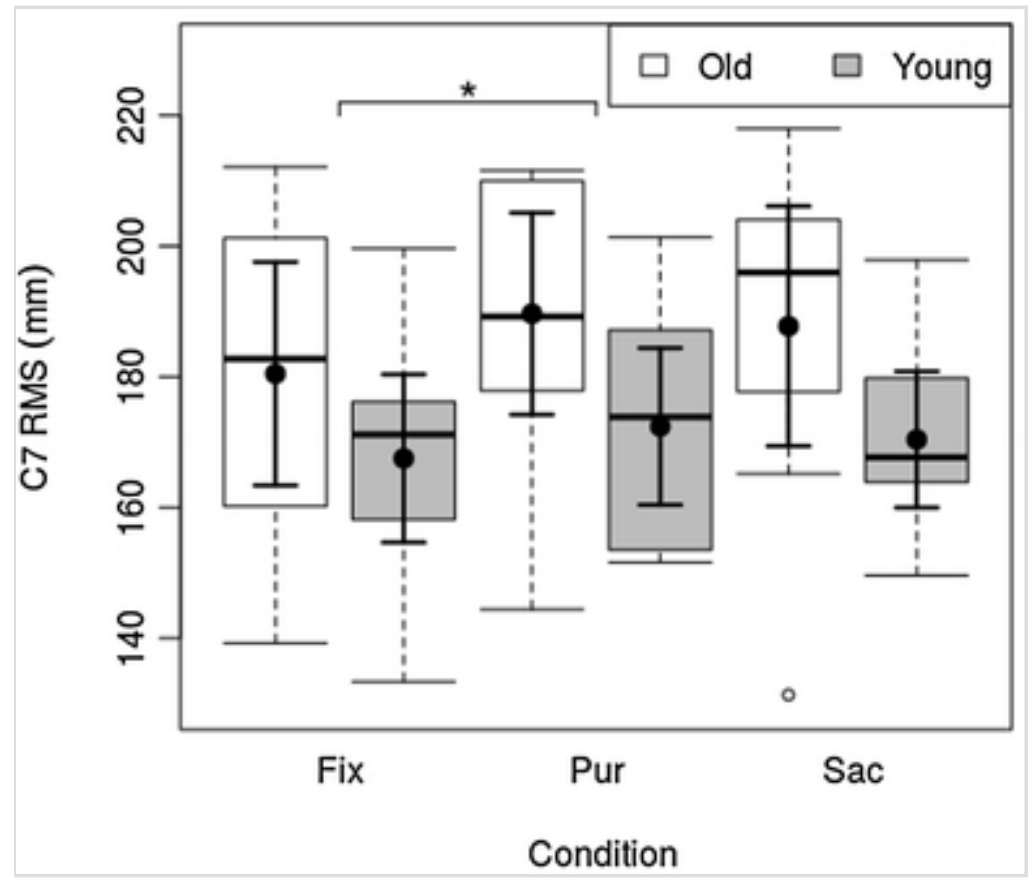

Fig. 3

Sacrum RMS in the ML direction in young $(n=10)$ and older $(n=10)$ participants during different eye movements. Fix stationary gaze fixation, Pur smooth pursuit, Sac Saccadice. Data are displayed as means and 95\% confidence intervals in bold, and medians and lower and upper quartiles with Tukey style whiskers (outliers plotted separately). Asterisk significant difference between conditions. Double asterisk significant difference between age groups

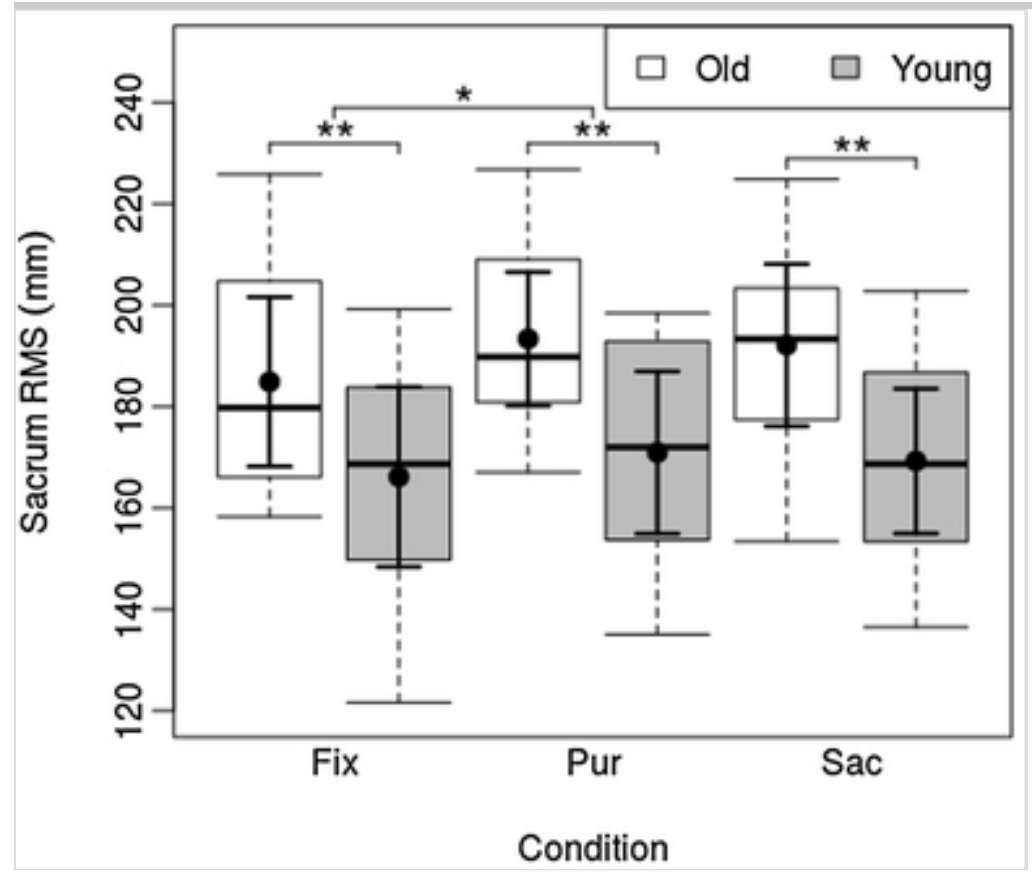


Sacrum RMS showed a main effect of condition $\left(F_{2,36}=5.06\right.$, $p=0.011$ ). Post hoc comparisons revealed larger Sacrum RMS during PUR compared to FIX ( $p=0.009, g_{\mathrm{av}}=0.27$ ), andbut no change for SAC relative to FIX. Sacrum RMS showed a main effect of age $\left(\mathrm{F}_{1,18}=5.05, p=0.037\right)$, with larger Sacrum RMS in the older group. Sacrum RMS showed no interaction effect between condition and age.

Trunk lean RMS showed no main effect of condition or age, or any interaction effect between condition and age.

\section{Lower limbs}

Mean step-width showed no main effect of condition or age, or any interaction effect between condition and age. Step-width CV (Fig. 4.) showed a main effect of condition $\left(F_{2,36}=4.75, p=0.049\right)$. Post hoc comparisons revealed larger step-width CV during PUR compared to FIX ( $p=0.052, g_{\mathrm{av}}=0.39$ ), andbut no change for SAC relative to FIX. Step-width CV showed a main effect of age $\left(F_{1,18}=5.08, p=0.037\right)$, with larger step-width CV in the older group. Step-width CV showed no interaction effect between condition and age.

Fig. 4

Step-width variability in young $(n=10)$ and older $(n=10)$ participants during different eye movement conditions. Fix stationary gaze fixation, Pur smooth pursuit, Sac saccadice. Data are displayed as means and 95\% confidence intervals in bold, and medians and lower and upper quartiles with Tukey style whiskers (outliers plotted separately). Asterisk significant difference between conditions. Double asterisk significant difference between age groups 


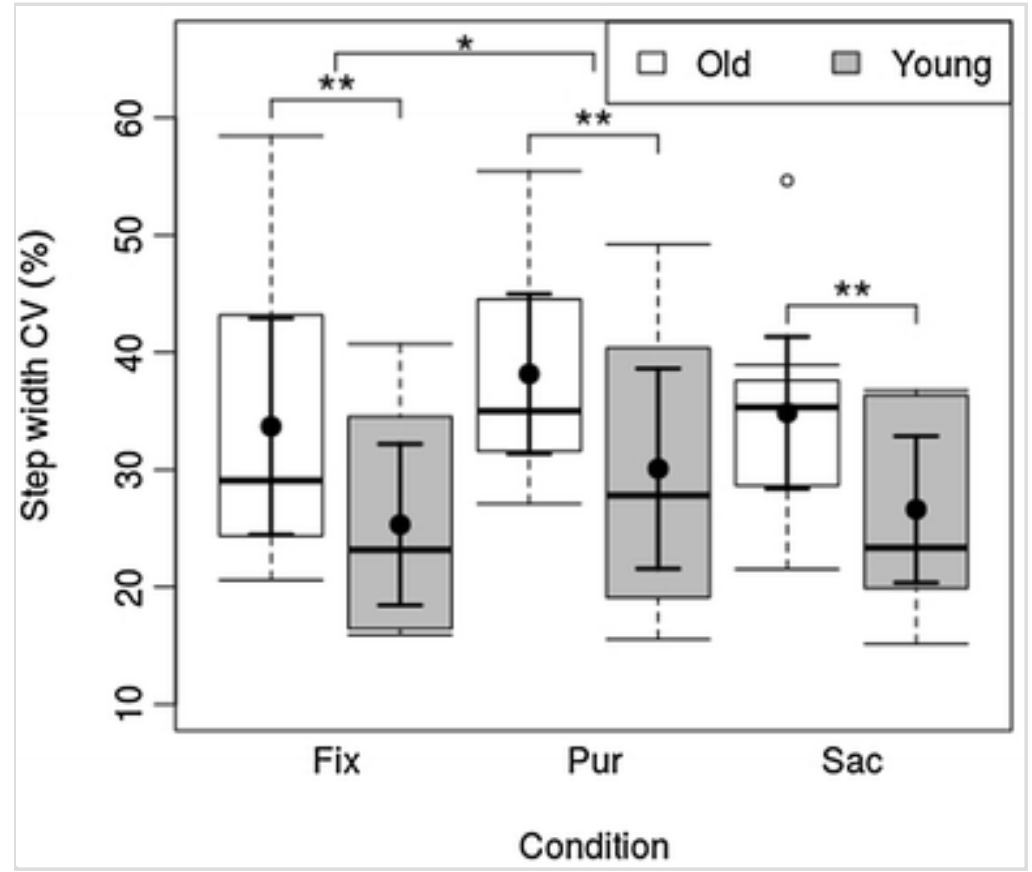

\section{Head rotation}

Head rotation showed no main effect of condition or age, or any interaction effect between condition and age. This suggests that all participants were able to follow the target with their eyes only and refrained from using head rotation in the direction of target movement.

\section{Gaze variables}

The correlation coefficients between the coordinate time series of the target and the gaze fixations, and gaze error RMS showed no main effect of condition or age, or any interaction effect between condition and age. The correlation analysis showed strong correlations $(r>0.8)$ in all conditions for all participants. These results indicate all of the participants followed instructions and completed the visual tasks aside from natural gaze errors.

\section{Discussion}

The present investigation assessed dynamic balance control during smooth pursuit and saccadic eye movements in young and older healthy females steady state walking. Smooth pursuits increased ML trunk movement and step-width variability compared to fixation of a 
stable target similarly in both age groups, although the elders demonstrated less baseline stability in all conditions. Whilst there were no changes for saccades relative to the stable target. The older females demonstrated greater ML trunk displacement and step-width variability throughout. However, despite the reduce baseline stability in the elders, the magnitude of change caused by smooth pursuits was similar in both age groups.

The present results support the hypothesis that visually tracking a moving target with smooth pursuits decreases balance control during walking. This was likely related to changes in retinal flow caused by the eye movements. Processing of optic flow for self-motion during eye movements is thought to occur in the medial superior temporal (MST) (Duffy and Wurtz 1991) and ventral intraparietal (VIP) areas of the visual cortex (Schaafsma and Duysens 1996). These regions have been linked to judging direction heading from optic flow (Zhang et al. 2004), and compensation for changes in the focus of expansion during smooth pursuits (Page and Duffy 1999). In the present experiment, the added complexity to optic flow caused by smooth pursuits would likely lead to increased processing demands within the MST and VIP areas, and this may have reduced visual sensitivity to self-motion.

Another factor which may have contributed to decreased balance during smooth pursuits is more complex extraocular signals. Extraocular signals have been shown to improve balance in standing humans, where small eye movements used to maintain gaze fixations during postural sway (initiated by the vestibulo-ocular reflex) provide information about body position relative to the fixation point (Guerraz and Bronstein 2008). During locomotion, fixation of a stable target would produce similar signals. For example, researchers found a minimum threshold of 0.3 degrees of eye movement for $1 \mathrm{~cm}$ of translational head movement was useful during standing (Guerraz and Bronstein 2008). During locomotion, the gait cycle would induce translation head movements meeting this threshold (Borg et al. 2015). It is thus likely that the extraocular component of balance control persists during walking, and if so, it is also likely that during a smooth 
pursuit, the eye rotation required to keep gaze fixated on the moving target would surpass any extraocular signals useful for balance control. This was also thought to occur during standing in previous experiments (Glaswer al. 2005; Laurens et al. 2010; Thomas et al. 2016).

In-support of the saccadic hypothesisSupporting the final hypothesis, there were no changes to balance during saccades compared to fixating a stable target. Because saccades are a series of fixations separated by rapid eye movements, and the target movement during SAC was completed in sub $20 \mathrm{~ms}$ and each saccade in 40-50 ms (Abrams et al. 1989), gaze was fixated on a stable target for the majority of the saccadic eye movement trials. Such conditions likely preserved the stable reference frame similar to FIX. It is also probable, therefore, that any element of extraocular postural stabilisation was also preserved. Even though the final displacement of the eye rotation during saccades was the same as during smooth pursuit, the nature of eye rotation and neural control to reach that displacement are different (Kowler 2011). In effect, the continuing rotation during smooth pursuits complicates extraocular signals, whilst the short rapid shifts of saccades preserve longer periods (pre and post-saccade) of fixation and thus useful extraocular signals.

Contrary to the ageing hypothesis, the negative change to balance during smooth pursuits was not more profound in the older adults. This is interesting since elders have previously been shown to have difficulties in interpreting optic flow for self-motion (Berard et al. 2009). However, comparing the present results with others is difficult since there have been no studies considering eye movements and balance in walking elders. One explanation is there was only a small effect (trunk: $g_{\mathrm{av}}=0.32$; lower limbs: $g_{\mathrm{av}}=0.39$ ) of smooth pursuits on balance in both age groups. The changes to retinal flow, therefore, may not have been profound enough to 'challenge' the ageing CNS enough to bring about a greater change. This would indicate that healthy elders are able to process visual flow for balance purposes during smooth pursuits as effectively as younger adults. However, the older adults demonstrated reduced balance throughout testing, with greater Sacrum 
displacement and step-width variability in all conditions. Thus, the elders were already at a disadvantage, which was probably due to a combination of musculoskeletal and sensory deficits which are considered normal in healthy ageing (Ambrose et al. 2013). Any further decrease to balance such as that shown in the present experiment is, therefore, certainly undesirable, particularly considering that greater baseline instability indicates a higher risk of falls (Ambrose et al. 2013). Further research is needed to assess whether smooth pursuits affect balance control in pathological ageing. For example, in patients with vestibular dysfunction and/or eye conditions such as peripheral vision loss-peripheral vision is more dominant in balance control (Guerraz and Bronstein 2008), and the loss of which may, therefore, exacerbate negative changes to balance control during smooth pursuits. Such research may be important for identifying those at increased risk of falls.

\section{Acknowledgements}

This research was funded by the Dowager Countess Eleanor Peel Trust. The authors would like to thank Aurora Leo and Michela Colli for their help during testing.

Compliance with ethical standards

Conflict of interest The authors declare that they have no conflicts of interest.

\section{References}

Abrams RA, Meyer DE, Kornblum S (1989) Speed and accuracy of saccadic eye movements: characteristics of impulse variability in the oculomotor system. J Exp Psychol Hum Percept Perform 15:529. doi:10.1037/0096-1523.15.3.529

Allison LK, Kiemel T, Jeka JJ (2006) Multisensory reweighting of vision and touch is intact in healthy and fall-prone older adults. Exp Brain Res 175:342-352. doi:10.1007/s00221-006-0559-7 
Ambrose AF, Paul G, Hausdorff JM (2013) Risk factors for falls among older adults: a review of the literature. Maturitas 75:51-61. doi:10.1016/j.maturitas.2013.02.009

Barre A, Armand S (2014) Biomechanical ToolKit: open-source framework to visualize and process biomechanical data. Comput Methods Programs Biomed 114:80-87. doi:10.1016/j.cmpb.2014.01.012

Bauby CE, Kuo AD (2000) Active control of lateral balance in human walking. J Biomech 33:1433-1440.

doi:10.1016/S0021-9290(00)00101-9

Berard JR, Fung J, McFadyen BJ, Lamontagne A (2009) Aging affects the ability to use optic flow in the control of heading during locomotion. Exp Brain Res 194:183-190.

doi:10.1007/s00221-008-1685-1

Birmingham E, Bischof WF, Kingstone A (2008) Gaze selection in complex social scenes. Vis Cogn 16:341-355.

doi:10.1080/13506280701434532

Borg O, Casanova R, Bootsma RJ (2015) Reading from a head-fixed display during walking: adverse effects of gaze stabilization mechanisms. PLoS One 10:e0129902.

doi:10.1371/journal.pone.0129902

Brach JS, Berlin JE, VanSwearingen JM et al (2005) Too much or too little step width variability is associated with a fall history in older persons who walk at or near normal gait speed. J Neuroeng Rehabil 2:1. doi:10.1186/1743-0003-2-21

\section{Bugnariu N, Fung J (2007) Aging and selective sensorimotor strategies in the regulation of upright balance. J Neuroeng Rehabil 4:1. doi:10.1186/1743-0003-2-21}

DeAngelis GC, Angelaki DE (2012) Visual-vestibular integration 
for self-motion perception. In: Murray MM, Wallace M (eds) The Nueral Basis of Multisensory Processes. CRC Press, Boca Raton AQ2

Dingwell J, Cusumano J, Cavanagh P, Sternad D (2001) Local dynamic stability versus kinematic variability of continuous overground and treadmill walking. J Biomech Eng 123:27-32. doi:10.1115/1.1336798

Dowiasch S, Marx S, Einhäuser W, Bremmer F (2015) Effects of aging on eye movements in the real world. Front Hum Neurosci 9:46. doi:10.3389/fnhum.2015.00046

Duffy CJ, Wurtz RH (1991) Sensitivity of MST neurons to optic flow stimuli. II. Mechanisms of response selectivity revealed by small-field stimuli. J Neurophysiol 65:1346-1359

Field A, Miles J, Field Z (2012) Discovering Statistics Using R. SAGE Publications, London

Foulsham T, Walker E, Kingstone A (2011) The where, what and when of gaze allocation in the lab and the natural environment. Vision Res 51:1920-1931. doi:10.1016/j.visres.2011.07.002

Franz JR, Francis CA, Allen MS et al (2015) Advanced age brings a greater reliance on visual feedback to maintain balance during walking. Hum Mov Sci 40:381-392.

doi:10.1016/j.humov.2015.01.012

Gibson JJ (1950) The perception of the visual world. Houghton Mifflin, Boston

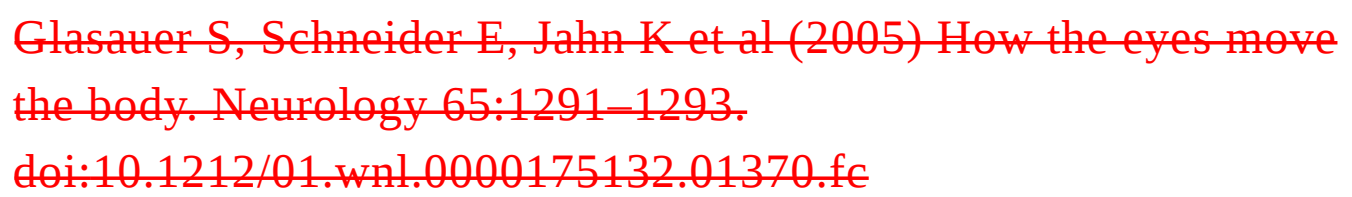

Guerraz M, Bronstein A (2008) Ocular versus extraocular control of 
posture and equilibrium. Neurophysiol Clin Neurophysiol 38:391-398. doi:10.1016/j.neucli.2008.09.007

Hallet PE (1986) Eye movements. In: Boff KR, Kaufman L, Thomas JP (eds) Handbook of human perception and performance. Wiley, New York, pp 10.1-10.112

\section{Hollman JH, Brey RH, Robb RA et al (2006) Spatiotemporal gait deviations in a virtual reality environment. Gait Posture 23:441-444. doi:10.1016/j.graitpost.2005.05.005}

Hollman JH, Brey RH, Bang TJ, Kaufman KR (2007) Does walking in a virtual environment induce unstable gait? An examination of vertical ground reaction forces. Gait Posture 26:289-294. doi:10.1016/j.gaitpost.2006.09.075

Hollman JH, Childs KB, McNeil ML et al (2010) Number of strides required for reliable measurements of pace, rhythm and variability parameters of gait during normal and dual task walking in older individuals. Gait Posture 32:23-28. doi:10.1016/j.gaitpost.2010.02.017

Kowler E (2011) Eye movements: the past 25years. Vision Res 51:1457-1483. doi:10.1016/j.visres.2010.12.014

Lakens D (2013) Calculating and reporting effect sizes to facilitate cumulative science: a practical primer for t-tests and ANOVAs. Front Psychol 4:863. doi:10.3389/fpsyg.2013.00863

Lappe M, Hoffmann K (2000) Optic flow and Eye Movements. Int Rev Neurobiol 44:29-47

Laurens J, Awai L, Bockisch C et al (2010) Visual contribution to postural stability: interaction between target fixation or tracking and static or dynamic large-field stimulus. Gait Posture 31:37-41. doi:10.1016/j.gaitpost.2009.08.241 
Lindemann U, Najafi B, Zijlstra W et al (2008) Distance to achieve steady state walking speed in frail elderly persons. Gait Posture 27:91-96. doi:10.1016/j.gaitpost.2007.02.005

Logan D, Kiemel T, Dominici N et al (2010) The many roles of vision during walking. Exp Brain Res 206:337-350.

doi:10.1007/s00221-010-2414-0

Logan D, Ivanenko YP, Kiemel T et al (2014) Function dictates the phase dependence of vision during human locomotion. $\mathrm{J}$

Neurophysiol 112:165-180. doi:10.1152/jn.01062.2012

McAndrew PM, Dingwell JB, Wilken JM (2010) Walking variability during continuous pseudo-random oscillations of the support surface and visual field. J Biomech 43:1470-1475. doi:10.1016/j.jbiomech.2010.02.003

Orendurff MS, Schoen JA, Bernatz GC, Segal AD (2008) How humans walk: bout duration, steps per bout, and rest duration. J Rehabil Res Dev 45:1077-1090. doi:10.1682/JRRD.2007.11.0197

Owings TM, Grabiner MD (2004) Variability of step kinematics in young and older adults. Gait Posture 20:26-29.

doi:10.1016/S0966-6362(03)00088-2

Page WK, Duffy CJ (1999) MST neuronal responses to heading direction during pursuit eye movements. J Neurophysiol 81:596-610

Paquette C, Fung J (2011) Old age affects gaze and postural coordination. Gait Posture 33:227-232.

doi:10.1016/j.gaitpost.2010.11.010

Paterson KL, Lythgo ND, Hill KD (2009) Gait variability in younger and older adult women is altered by overground walking protocol. Age Ageing 38:745-748. doi:10.1093/ageing/afp159 
Patla AE, Vickers JN (2003) How far ahead do we look when required to step on specific locations in the travel path during locomotion? Exp Brain Res 148:133-138.

doi:10.1007/s00221-002-1246-y

Peirce JW (2007) PsychoPy—psychophysics software in Python. J Neurosci Methods 162:8-13. doi:10.1016/j.jneumeth.2006.11.017

Royden CS, Crowell JA, Banks MS (1994) Estimating heading during eye movements. Vision Res 34:3197-3214.

doi:10.1016/0042-6989(94)90084-1

Schaafsma S, Duysens J (1996) Neurons in the ventral intraparietal area of awake macaque monkey closely resemble neurons in the dorsal part of the medial superior temporal area in their responses to optic flow patterns. J Neurophysiol 76:4056-4068

Schellenbach M, Lövdén M, Verrel J et al (2010) Adult age differences in familiarization to treadmill walking within virtual environments. Gait Posture 31:295-299.

doi:10.1016/j.gaitpost.2009.11.008

Thomas NM, Bampouras TM, Donovan T, Dewhurst S (2016) Eye movements affect postural control in young and older females. Front Aging Neurosci 8:216. doi:10.3389/fnagi.2016.00216

Warren WH, Hannon DJ (1990) Eye movements and optical flow. J Opt Soc Am A 7:160-169

Warren WH, Kay BA, Yilmaz EH (1996) Visual control of posture during walking: functional specificity. J Exp Psychol Hum Percept Perform 22:818-838. doi:10.1037/0096-1523.22.4.818

\section{Wass E, Taylor NF, Matsas A (2005) Familiarisation to treadmill walking in unimpaired older people. Gait Posture 21:72-79. doi:10.1016/j.gaitpost.2004.01.003}


Yeh TT, Cluff T, Balasubramaniam R (2014) Visual reliance for balance control in older adults persists when visual information is disrupted by artificial feedback delays. PLoS One 9:1-9. doi:10.1371/journal.pone.0091554

Zeni J, Richards J, Higginson J (2008) Two simple methods for determining gait events during treadmill and overground walking using kinematic data. Gait Posture 27:710-714.

doi:10.1016/j.gaitpost.2007.07.007

Zhang T, Heuer HW, Britten KH (2004) Parietal area VIP neuronal responses to heading stimuli are encoded in head-centered coordinates. Neuron 42:993-1001. doi:10.1016/j.neuron.2004.06.008

${ }^{1}$ In the present manuscript, the term 'balance control' defines maintenance of an upright posture during locomotion. 